\title{
Reconstruction of a Worn or Mutilated Dentition with the Utilization of an over Denture Appliance for a Patient with Enamel Deficiency - A Case Report
}

\author{
Klenise Silva Paranhos*, Harprit Kanda, Michael Ghalili and Philip Kotick \\ Adjunct Clinical Instructor, Cariology and Comprehensive Care Cariology, USA \\ Received: July 07, 2017; Published: July 18, 2017 \\ *Corresponding author: Klenise S Paranhos, Rambling Brook Road, Chappaqua, New York 10514, Email: kp21@nyu.edu
}

\begin{abstract}
Background: Evidence suggests that the retention of mandibular roots for the preservation of bone in patients by utilizing an over denture is a conservative approach for a fixed and removable prosthetic reconstruction.

Case description: A young adult with worn and fractured dentition exhibited severe loss of function and esthetics, exhibiting both occlusal discrepancies as well as esthetic problems. The treatment was preventive as well as conservative in nature. It was recommended to preserve endodontically treated teeth in order to preserve the bone in young patients with a severely worn, non-restorable dentition. By reviewing the current literature, several aspects of patient selection and treatment planning are discussed.
\end{abstract}

Practical Implications: This case report suggests that patients can benefit from a fixed and removable maxillary and mandibular prosthetics by using over denture stud attachments, preserving the endodontically treated roots in order to maintain bone.

Keywords: Enamel Hypoplasia; Over denture; Overlays; Stud Attachment

\section{Introduction}

The technique of retaining roots and utilizing an over denture in patients with worn and non-restorable teeth is not a new approach $[1,2]$. When teeth are extracted, the residual alveolar bone resorbs and continues to resorb over time [3]. The alveolar resorption is greatest in the first months following extraction of teeth, depending upon biological and mechanical factors. However, the rate of resorption on the mandible is faster compared with the maxilla [4]. In addition, greater bone loss was found in the anterior part of the jaws, especially in the mandible [4]. Resorption of the maxilla is apically and poster apically while the mandible is apically and anteroapically. Consequently, if teeth or roots could be maintained in the anterior region of the mandible, it would be a significant benefit to the patient though bones preservation. Prostheses supported by stud type attachments over roots provide a retentive over denture, which is more stable.

The use of an over denture attachment system for a partial/ complete removable prosthesis in a patient with endodontically treated retained roots is a predictable approach. The attachment system provides a balanced solution between functional stability and preservation of roots, maintenance of the alveolar bone, cosmetic appeal and economics. Due to the amount of caries and the condition of this young patient's enamel, the treatment option of choice was to preserve the roots of the mandibular teeth. This clinical report describes the placement of an over denture onto endodontically treated abutments utilizing stud attachments. Lithium disilicate ceramic on lays and/or full coverage restorations on the remaining maxillary teeth were utilized to restore the anatomical tooth structure and vertical dimension of occlusion. Full coverage restorations were placed on the maxillary teeth, premolar to premolar, in order to restore the maxillary teeth.

\section{Clinical Case Report}

A 22-year-old patient presented to the International Comprehensive Program at New York University College of Dentistry with the chief complaint that his teeth keep fracturing and he also did not like their appearance. He wanted them restored in order to improve his smile (Figure 1). He also exhibited halitosis, closed vertical dimension of occlusion, xerostomia and a history of bruxism. During the review of the patient's medical history, we were advised of the use of hormone replacement therapy for transgender (female-to-male) treatment that consists of androgens like testosterone. This therapy may also contribute to this teenage patient's oral conditions, generalized hypoplasia of the enamel; by 
due to an increase caries risk [5-7]. Others side effects include Oral Candidiasis, Oral Hairy Leukoplakia, Aphthous Stomatitis [6-8]. Radiographic examination confirmed that teeth \#19, 20, 21, 24, 25, $26,27,28,29$ and 30 were endodontically treated and showed an impacted maxillary canine with an orthodontic chain to access force eruption remained (Figure 2). The patient abandoned orthodontic therapy about six years ago.

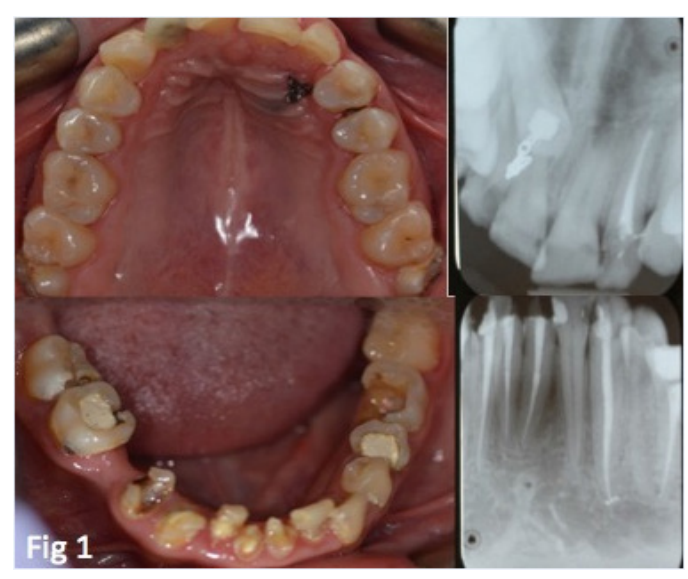

Figure 1

Physical examination of the head and neck region revealed within normal limits. Intra oral clinical findings revealed poor oral hygiene and generalized caries in all mandibular and maxillary teeth. Periodontal evaluation indicated gingivitis and probing depth measurements from 1-3 mm with no presence of clinical attachment loss. Several treatment options were presented taking into consideration the patient's age and the fact many of his mandibular teeth were endodontically treated. Retention of the roots of the mandibular teeth is desirable to preserve the bone. The stud attachment system consisted of:

i. Metal post with a ball that is cemented into the root.

ii. Metal housing with nylon inserted that is incorporated into the removable prosthesis.

After the post is cemented, the nylon cap is inserted and threaded into the metal keeper. Then, the nylon cap and metal keeper system are incorporated within over denture with auto polymerizing acrylic resin. Patient was advised that if the nylon cap needs a replacement, it is an in-office procedure.

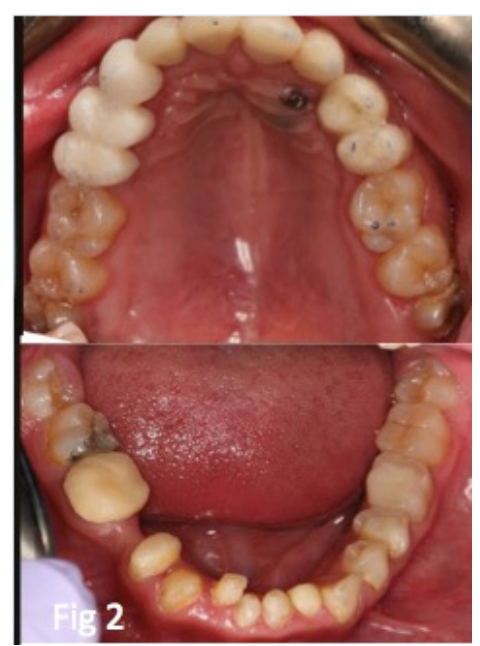

Figure 2

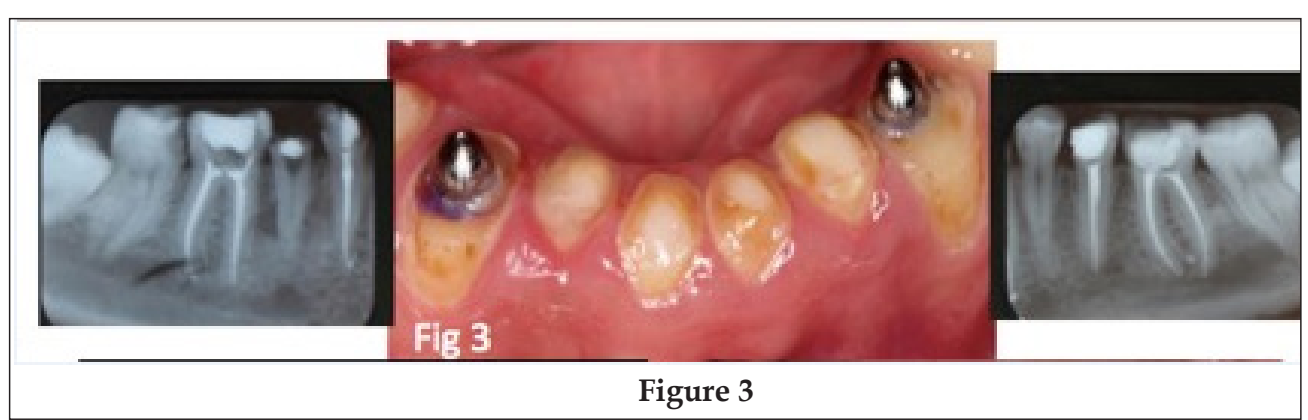

Stud attachments were used on teeth \#22 and \#27 since these teeth had longer roots and thus more encased in bone as compared to the incisors. A post \& core preparation and provisionalization was also performed on tooth \#30. Endodontic therapy was performed on tooth \#23, which had a deep carious lesion. The mandibular anterior teeth were restored with composite resin to cover the access channels (Figure 3). A mandibular transitional removable partial denture was fabricated to restore the vertical dimension covering the roots of teeth \#19, \#20 and \#22 - \#27 and replacing tooth \#29.

Several adjustments were made to fit the removable partial denture over the retained roots and ensure the patient's phonetics and function. Stud attachments were placed in to teeth, \#22 and \#27 (Figure 4). The adjustment of the transitional partial denture was altered in order to fit over the stud attachments. The patient was very happy and comfortable with the result. The day the transitional partial denture was inserted, the patient had a job interview that led to his first employment opportunity. For fabrication of the final mandibular over denture a new impression and occlusal registration was made according to the restored vertical dimension. Lithium disilicate ceramic onlays were placed on \#18, \#21, \#30 and \#31 in order to restore the occlusion (Figure $5)$. 


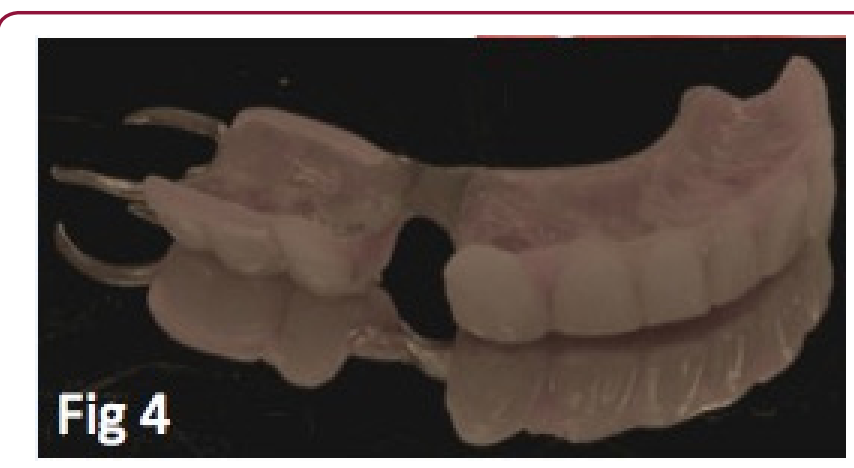

Figure 4

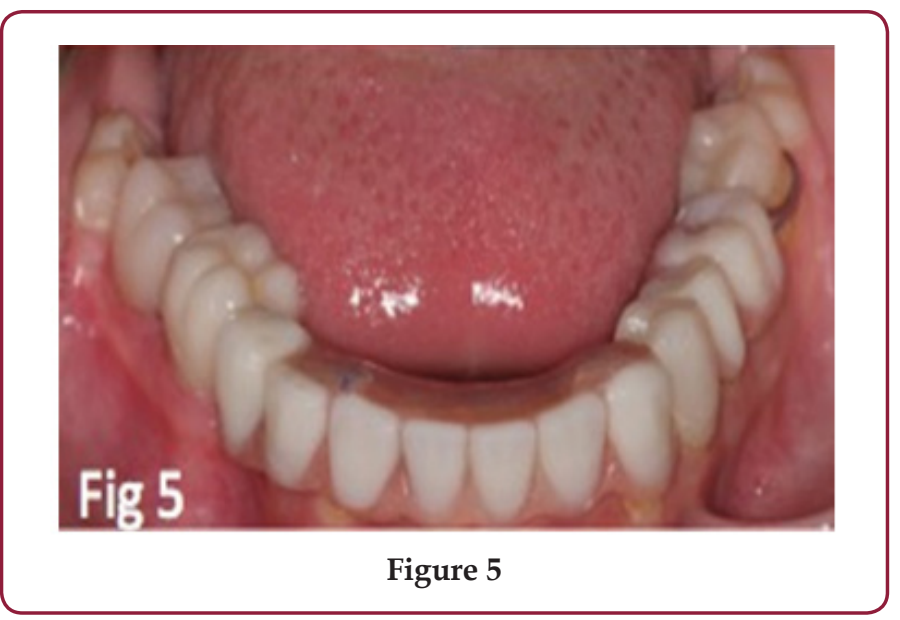

The placement and luting of the male keeper on the definitive over denture was accomplished with auto polymerizing methacrylate pink acrylic auto polymerization. The definitive over denture was delivered after occlusal adjustments were completed. Teeth \#4, \#5, \#7, \#8, \#9, \#10, \#11, \#12 and \#13 had generalized rampant caries extend to all teeth surfaces and were prepared for full coverage restoration and provisionalized using individual auto polymerized acrylic resin crowns to re- establish the vertical dimension (Figure 6A). Consequentially porcelain crowns were fabricated on teeth \# 5, \# 7-13 (Figure 6B). The patient was advised to have teeth \# 1, 6, 16, 17 and 32 extracted by an oral surgeon. Preventive considerations and nutritional dietary consultation was offered by way of recommending a sugar free diet and comprehensive oral hygiene. Regular topical fluoride application procedures were advised to use as a cavity-prevention strategy as well as use of a high concentrated fluoride gel. This treatment plan was considered the preferred option for this patient due to a prior lack of initiative from the patient to take steps regarding his teeth and also his financial circumstances. Because of the amount of caries and the condition of the patient's enamel, full coverage restorations were an appropriate choice. They accomplished the following:

i. Restoration of the teeth,

ii. Restoration of the vertical dimension of occlusion and

iii. Alteration of both the shape and shade of the patient's teeth.

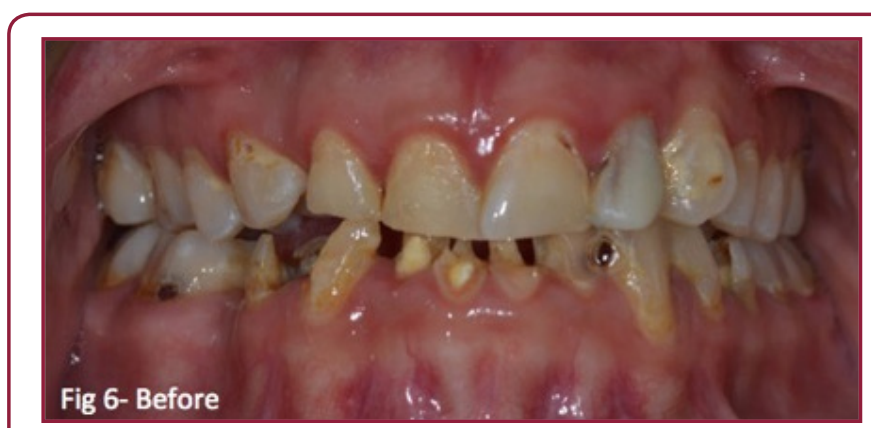

Figure 6A

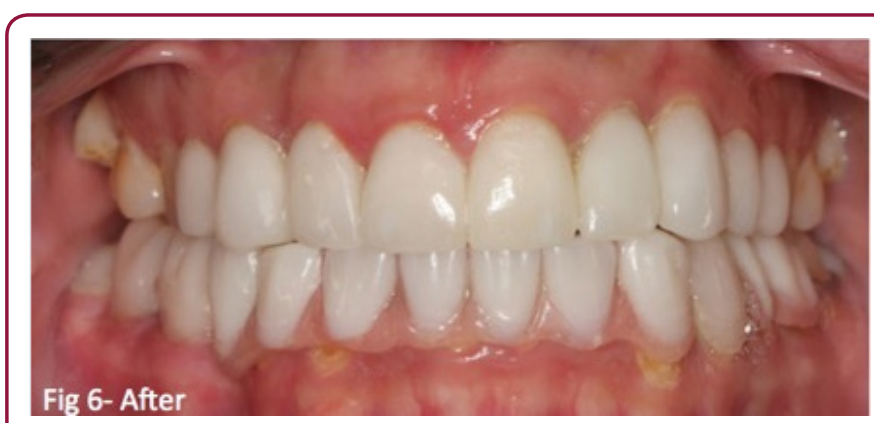

Figure 6B

\section{Discussion}

The association between worn teeth and emotional status relating to psychosocial impact of oral health is a major concern especially in young patients. When this association includes the combination of hormone replacing therapy transgender man (female-male), this intensifies the emotional status, general and oral health and the risk of caries [5]. The parents and oral health professionals play a vital role in the care of this young patient providing necessary and preventive care to avoid the risks of relapse. The oral health provider must make an assessment of each patient's competence and ability to understand and importance of theirs participation during the process. The presence of dental caries appears rapidly in cases of enamel hypo mineralization and hyperplasia. Most of the time it is not possible to differentiate the two types on clinical manifestation alone. Hypo mineralization is a decrease in the mineral content of the enamel that leads to "soft" enamel [9]. These characteristics create enamel, which is more opaque and/or whiter. The relationship between hypo mineralization and dental caries progression is affected by many factors such as poor oral hygiene, tobacco use, alcohol consumption, xerostomia and high intake of dietary carbohydrates. In many cases, environmental factors that produce injury to ameloblasts (nutritional deficiency, especially vitamin A,C,D; exanthematous diseases (eg: measles, chicken pox, scarlet fever), congenital syphilis, hypocalcaemia, birth injury, prematurity, local injury or trauma and idiopathic causes [10]. The enamel appears more susceptible to injury than normal.

Enamel hypoplasia is a defect in the enamel that results in less quantity of enamel than normal $[5,11,12]$. The defect can be a small pit or dent in the tooth or can be so widespread that the entire tooth is small and/or mis-shaped. This type of defect may cause tooth 
sensitivity, may be unsightly or may be more susceptible to dental caries. Some genetic disorders, for example hypocalcaemia, cause all the teeth to have enamel hyperplasia [6]. Enamel hyperplasia can occur on any tooth or on multiple teeth $[13,14]$. The teeth may appear white, yellow or brownish in color with a rough or pitted surface. In some cases, the quality of the enamel is affected as well as the quantity. Environmental and genetic factors that interfere with tooth formation are thought to be responsible for enamel hyperplasia $[5,6,10,15]$. This includes trauma to the teeth and jaws, intubation of premature infants [15], infections during pregnancy or infancy, poor pre-natal and post-natal nutrition, hypoxia, exposure to toxic chemicals and a variety of hereditary disorders [16]. Frequently, the cause of enamel hyperplasia in a particular patient is difficult to determine. It is common in permanent dentition even if no previous report describes these characteristics in the patient's primary teeth.

The earlier diagnosis of low mineral content of the enamel may be a valuable screening tool in determining the higher than average caries risk. Prevention before development of caries is the ideal and predictable treatment by applying sealants, topic and systemic fluoride treatment during prophylactic office visits. Treatment options depend on the severity of the disease on a particular tooth and the symptoms associated with it. The most conservative treatment consists of bonding the tooth [17] to protect it from further wear or sensitivity. In some cases, the enamel destruction prevents formation of an acceptable bond. Therefore, we have to use less conservative treatment options [18,19], which include use of full coverage restorations or extraction of affected teeth and replacement with a complete or partial removable denture, a fixed partial prosthesis or implant. Note that, treatment of teeth with enamel hyperplasia must be determined on an individual basis. It is important to mention that the early clinical diagnosis of the anomalies of enamel is extremely important because it can consequentially increase risk for caries susceptibility, increased wear, tooth sensitivity and poor aesthetics. Identifying predisposing factors might encourage preventive methods.

\section{Conclusion}

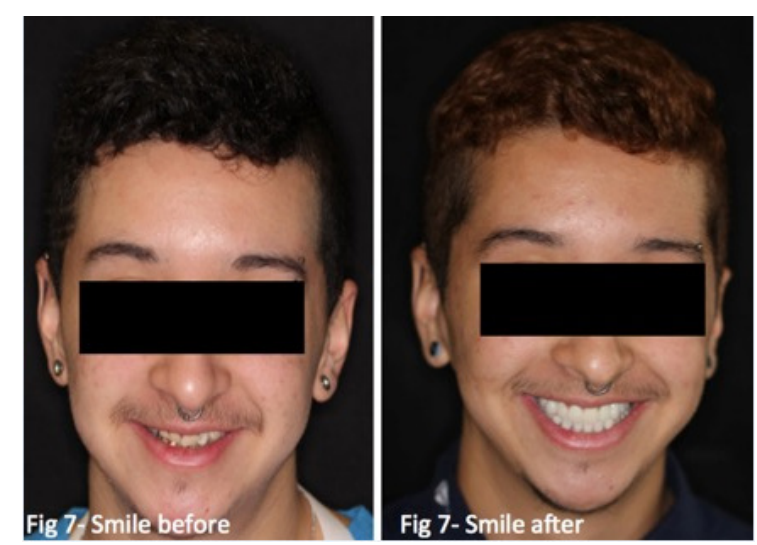

Figure 7

This case report is an example of how to achieve patient satisfaction and motivation by selection of the appropriate treatment plan. The priority is the patient's change in smile, function and phonetics before and after provisionalization. There was a change in shade and shape of the teeth as well, which improved both patient's appearance and speech. The retention of the mandibular roots in order to preserve the bone in this young patient, increase his confidence and home care of his teeth. This major change has made him confident to smile again and re-join society as a contributing member in his preferred career.

\section{References}

1. Brewer AA, Morrow RM (1980) Over dentures $2^{\text {nd }}$ edn., St. Louis The CV Mosby Co. pp: 347.

2. Fenton AH (1998) The Decade of Overdentures:1970-1980 J Prosthet Dent 79(1): 31-36.

3. Atwood DA, Coy WA (1971) Clinical, Cephalometric and Densitometric Study of Reduction of Residual Ridges. J Prosthet Dent 26(3): 280-295.

4. Tallgren A (1972) The Continuing Reduction of the Residual Alveolar Ridge in Complete Denture Wearers: a Mixed Longitudinal Study Covering 25 years. J Prosthet Dent 27(2): 120-132.

5. Manson JE, Allison MA, Rossouw, Carr J, Langer RD, Hsia J, et al. (2007) Estrogen therapy and coronary-artery calcification. N Engl J Med 356(25): 2591-2602.

6. Wierckx K, Mueller S, Weyers S, Van Caenegem E, Roef G, et al. (2012) Long-term evaluation of cross-sex hormone treatment in transsexual persons. J Sex Med 9(10): 2641-2651.

7. Ghom Govindrao Anil (2010) Textbook of oral medicine and radiology. $2^{\text {nd }}$ edn., Jaypee: New Delhi pp: 533-556.

8. Shafer, Levy, Hine (2005) Textbook of Oral Pathology. $5^{\text {th }}$ edn., Elsevier: New Dellhi pp: 35-44.

9. Weerheijm KL, Jalevik B, Alaluusua S (2001) Molar-Incisor hypomineralization. Caries Res 35(5): 390-391.

10. R. Rajendran Shafer's Textbook Of Oral Pathology.

11. layton RL, Warren JJ, Kanellis MJ, Levy SM, Islam M (2001) Prevalence of enamel hypoplasia and isolated opacities in the primary dentition. Pediatric Dentistry 23(1): 32-36.

12. Witkop CJ (1988) Amelogenesis imperfecta, dentinogenesis imperfecta and dentin dysplasia revisited: problems in classification. J Oral Pathol 17(9-10): 547-553.

13. Seow WK (1991) Enamel hypoplasia in the primary dentition: a review. ASDC J Dent Child 58(6): 441-452.

14. Rugg-Gunn AJ, Al Mohammadi SM, Butler TJ (1998) Malnutrition and developmental defects of enamel in 2- to 6-year-old Saudi boys. Caries Res 32(3): 181-192.

15. Slayton RL, Warren JJ, Kanellis MJ, Levy SM, Islam M (2001) Prevalence of enamel hypoplasia and isolated opacities in the primary dentition. Pediatric Dentistry 23(1): 32-36.

16. Brook AH, Fearne JM, Smith J (1997) Environmental causes of enamel defects. Ciba Foundation Symposium 205: 212-221.

17. Li RW (1999) Adhesive solutions: report of a case using multiple adhesive techniques in the management of enamel hypoplasia. Dent Update 26(7): 277-287.

18. Quinonez R, Hoover R, Wright JT (2000) Transitional anterior esthetic restorations for patients with enamel defects. Pediatric Dent 22(1): 6567.

19. Miller PA (1958) Complete Dentures Supported by Natural Teeth. J Prosthet Dent 8: 924-928. 


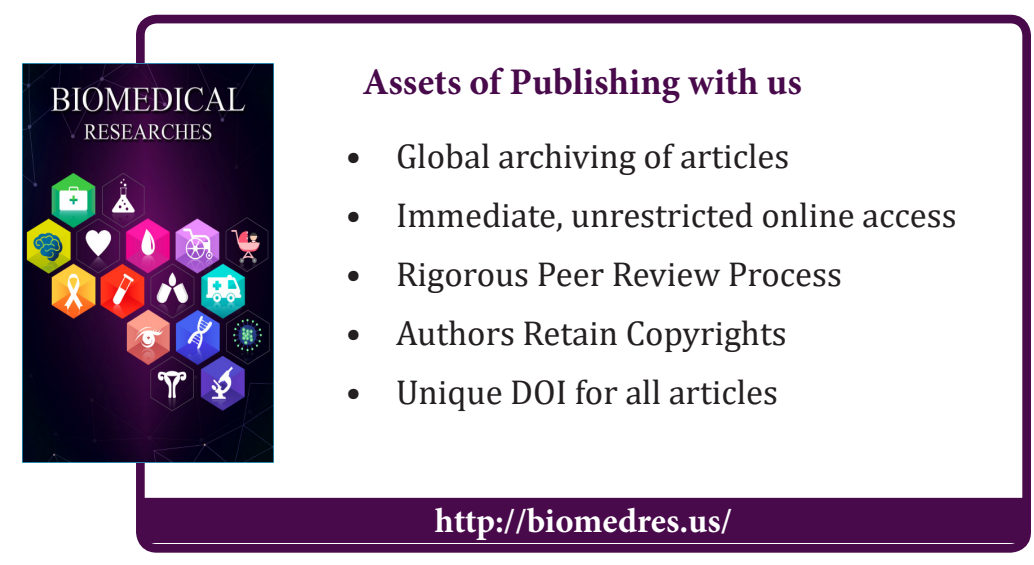

